\title{
The research on the hydraulic numerical simulation of flux limiter function
}

\author{
Dawei Sun ${ }^{1, a}$, Bingxi Sun ${ }^{1}$ \\ ${ }^{1}$ College of Physics and Electronic Information, Inner Mongolia University for the Nationalities, \\ Tongliao, Inner Mongolia , 028000, China \\ a'weidasun@163.com
}

Keywords: TVD flux limiter function, discontinuity catch, MUSCL form.

\begin{abstract}
The advantages of the form of this paper is numerical calculation without spurious numeric, and keeps monotonicity of numerical value. It contrasts the difference among various flux limiter flux limiter function in solving equations by examples and verifies the advantages which is conservative, robustness, spurious numericalosc and high-resolution catches discontinuity when introducing TVD flux limiter function Finite Volume Method model in numerical simulation.
\end{abstract}

\section{Introduction}

The advantages of TVD form is great keeping the monotonicity of numerical solution[1], efficiently weakening numerical osc which produces in cell boundaries, having high solution to discontinuity. But its disadvantage is the precision of TVD form can reach second order at most, and it only has first order at local extremum, which makes extreme value attenuation. And there isn't super-first order TVD form on multidimensional problems[2].

This paper is based on Roe's approximate Riemann solution[3], gets high-order accuracy shallow water equations discrete form by reconstitution and flux limiter function, and compares the merits and demerits in solving equations of flux limiter function in solving equations by examples.

\section{The discretization of the governing equations}

\subsection{Governing equation and numerical discretization}

The conservation form of vertically averaged two-dimension governing equation,

in the equation,

$$
\frac{\partial U}{\partial t}+\frac{\partial f}{\partial x}+\frac{\partial g}{\partial y}=S
$$

$$
U=\left[\begin{array}{c}
H \\
H u \\
H v
\end{array}\right] f=\left[\begin{array}{c}
H u \\
H u^{2}+\frac{g H^{2}}{2} \\
H u v
\end{array}\right] g=\left[\begin{array}{c}
H v \\
H u v \\
H v^{2}+\frac{g H^{2}}{2}
\end{array}\right] S=\left[\begin{array}{c}
0 \\
g H\left(-S_{o x}-S_{f x}\right) \\
g H\left(-S_{o y}-S_{f y}\right)
\end{array}\right]
$$

where $\mathrm{H}$ is the total water depth, $u$ and $v$ are the vertically integrated velocities in $X$ and $Y$ directions; $g$ is the acceleration of gravity; $S_{o x}=\partial Z_{b} / \partial x$ and $S_{o y}=\partial Z_{b} / \partial y$ are the bottom slope source items in $\mathrm{X}$ and $\mathrm{Y}$ directions; $Z_{b}$ is the bottom elevation relative to still water surface; $S_{f x}=n^{2} u \sqrt{u^{2}+v^{2}} H^{-4 / 3}$ and $S_{f y}=n^{2} v \sqrt{u^{2}+v^{2}} H^{-4 / 3}$ are the bottom frictional resistance source items in $\mathrm{X}$ and $\mathrm{Y}$ directions; $\mathrm{n}$ is the Manning coefficient.

Have $F=(f, g)$, the vector form(1) can be simplified into equation (2),

$$
U_{t}+\nabla \cdot F=S
$$


in the equation, $\nabla=\left(\frac{\partial}{\partial x}, \frac{\partial}{\partial y}\right)$.

The equation(2) was integrated in cell $\Omega_{i}$,

$$
\int_{\Omega_{i}} \frac{\partial U}{\partial t} d \Omega_{i}+\int_{\Omega_{i}} \nabla \cdot F d \Omega_{i}=\int_{\Omega_{i}} S d \Omega_{i}
$$

if the conservative variable $U_{i}$ set in cell-center, based on the Green formula, equation(3) from surface integral into line integral which is composed of cell $\Omega_{i}$ boundaries.

$$
\frac{\Delta U_{i}}{\Delta t}\left|\Omega_{i}\right|=-\oint_{l} F \cdot n d l+\int_{\Omega_{i}} S d \Omega_{i}
$$

where $l$ is the control unit boundaries; $n=\left(n_{x}, n_{y}\right)=(\cos \theta, \sin \theta)$ is the numerical flux in outer normal direction at cell boundaries; $\theta$ is the included angle between outer normal vector and $\mathrm{x}$-axis forward direction; $\left|\Omega_{i}\right|$ is the cell area.

The left of equation(4) can be discretized by line integral,

$$
\Delta U_{i}=-\frac{\Delta t}{\left|\Omega_{i}\right|} \sum_{j=1}^{3}\left(F_{i j}^{*} \cdot n_{i j}\right) l_{i j}+\frac{\Delta t}{\left|\Omega_{i}\right|} \int_{\Omega_{i}} S d \Omega_{i}
$$

where $F_{i j}^{*} \cdot n_{i j}$ is the normal numerical flux in $j$ line, $l_{i j}$ is the side length of $j$ line.

$$
\text { when } \bar{S}_{i}=\frac{1}{\left|\Omega_{i}\right|} \int S d \Omega \text {, }
$$

$$
\Delta U_{i}=-\frac{\Delta t}{\left|\Omega_{i}\right|} \sum_{j=1}^{3}\left(F_{i j}^{*} \cdot n_{i j}\right) l_{i j}+\Delta t \bar{S}_{i}
$$

\subsection{The numerical flux of Roe scheme}

After integral averaged, the conservative variable in every control unit is constant normally distributed that forms the regimentation function state. According to the Riemann problem with column, in every control unit boundaries, variable is considered discontinuity. If time interval is short enough, and each control unit boundaries doesn't have influence on neighboring cells flux, so the numerical flux is considered to be unequal. It shows respectively by $U_{i, L}, U_{i, R}$.

$$
F_{i, j}=F^{*}\left(U_{i, L}, U_{i, R}\right)
$$

The numerical flux of Roe scheme:

$$
F^{*} \cdot n=\frac{1}{2}\left[(f, g)_{R} \cdot n+(f, g)_{L} \cdot n-|\tilde{J}|\left(U_{R}-U_{L}\right)\right]
$$

where $U_{L}, U_{R}$ is the conservative variable on both side of the interface; $\tilde{J}$ is Roe mean Jocobian matrix;

$$
\tilde{J}=\frac{\partial(F \cdot n)}{\partial u}=\frac{\partial f}{\partial U} n_{x}+\frac{\partial g}{\partial U} n_{y}=\left[\begin{array}{ccc}
0 & n_{x} & n_{y} \\
\left(c^{2}-u^{2}\right) n_{x}-u v n_{y} & 2 u n_{x}+v n_{y} & u n_{y} \\
-u v n_{x}+\left(c^{2}-v^{2}\right) n_{y} & v n_{x} & u n_{x}+2 v n_{y}
\end{array}\right]
$$

where $C=\sqrt{g h}$ is wave velocity.

$$
\tilde{u}=\frac{u_{R} \sqrt{H_{R}}+u_{L} \sqrt{H_{L}}}{\sqrt{H_{R}}+\sqrt{H_{L}}}, \tilde{v}=\frac{v_{R} \sqrt{H_{R}}+v_{L} \sqrt{H_{L}}}{\sqrt{H_{R}}+\sqrt{H_{L}}}, \tilde{c}=\sqrt{g \frac{\left(H_{R}+H_{L}\right)}{2}}
$$

where $u_{L}, u_{R}$ is the $\mathrm{X}$ direction flow velocity on the both side of cell boundaries; $v_{L}, v_{R}$ is the $\mathrm{Y}$ direction flow velocity on the both side of cell boundaries; $H_{L}, H_{R}$ is the depth of water on the both side of cell boundaries. 
This paper uses the Hancock preparative estimation and rectification two-step scheme[4].

(1) The preparative estimation step:

(2) The rectification step:

$$
\bar{U}_{i}^{n+1 / 2}=\bar{U}_{i}^{n}-\frac{1}{2} \frac{\Delta t}{\left|\Omega_{i}\right|} \sum_{j=1}^{3}\left(F_{1 i j}^{*} \cdot n_{j}\right) \Delta l_{j}+\Delta t \bar{S}_{i}^{n}
$$

$$
\bar{U}_{i}^{n+1}=\bar{U}_{i}^{n+1 / 2}-\frac{\Delta t}{\left|\Omega_{i}\right|} \sum_{j=1}^{3}\left(F_{1 i j}^{*} \cdot n_{j}\right) \Delta l_{j}+\Delta t \bar{S}_{i}^{n+1 / 2}
$$

where $\bar{U}_{i}^{n+1 / 2}$ is intermediate variable.

\section{High precision form}

The important procedure of finite volume method spatial dispersion is reconsitution of controlling unit boundaries conservative variable. It relates to the model space precision and resolution. After measuring comprehensively, this paper adopts MUSL form to reconsitution numerical flux in boundaries.

\subsection{TVD flux limiter function}

The conservative variable $U_{i}$ and $U_{i+1}$ on each side of interface is replaced by $U_{i+1 / 2}^{L}$ and $U_{i+1 / 2}^{R}$, the space computational accuracy of this method is second order. Where the surface gradient is great can produces numerical value spurious numericalosc and make calculation error, this paper introduce TVD method to avoid this situtation.

Three types of TVD flux limiter function as shown below:

$$
\begin{gathered}
\text { Roe's Superbee: } \phi(r)=\max (0, \min (2 r, 1), \min (2, r)) \\
\text { Roe's Minmod }: \phi(r)=\max (0, \min (1, r))
\end{gathered}
$$

Chakravarthy \&Osher : $\phi(r)=\max (0, \min (r, \sigma)), 1 \leq \sigma \leq 2$

$$
\text { MUSCL }: \phi(r)=\frac{(r+|r|)}{(1+|r|)}
$$

where $\mathrm{r}$ is the conservative variable variation ratio of neighboring control units. For second order dispersed precision of governing equation, and the sufficient condition of TVD property is the choice of flux limiter function $\phi(r)$ in the shadow region, as shown in Fig.1, broken line 1 is Superbee flux limiter function, curve2 is MUSCL flux limiter function, broken line 3 is Minmod flux limiter function ,broken line 4 is Osher flux limiter function.

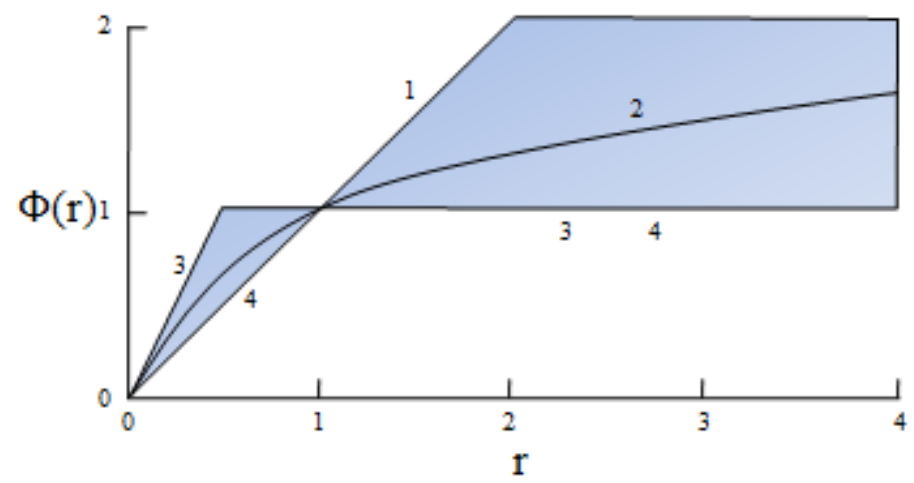

Fig.1

By computing the instance of one-dimensional Leveque water wave travel, and compared with experiment to verify the form in this paper have high precision, robustness and spurious numericalosc in the condition of varied topography. Leveque first proposed in document[5], the model is equally wide rectangle cross watercourse of 1 meter in length, the bottom elevation is:

$$
Z_{b}=\left\{\begin{array}{cc}
0.25[\cos (\pi(x-0.5) / 0.1] & |x-0.5| \leq 0.1 \\
0.1 & \text { else }
\end{array}\right.
$$


Initial flow velocity $\mathrm{u}=0$, initial water level:

$$
h(x)=\left\{\begin{array}{cc}
1.0+\varepsilon & 0.1<x<0.2 \\
1.0 & \text { else }
\end{array}\right.
$$

where $\varepsilon=0.01$, the number of grid cell is 200 . Simulation $\mathrm{t}=0.2 \mathrm{~s}$ water level and flow velocity as shown in fig.4. From Fig.4, even at the bottom altitude high-variability, the process of water level and flow velocity are very smooth, which shows the model is of adaptation to complex topography computational domain, but the model without flux limiter function and cell boundaries reconstruction has not work so well, shows under the complex topography condition, it's very essential introduces Riemann discontinuous solution numerical scheme based .
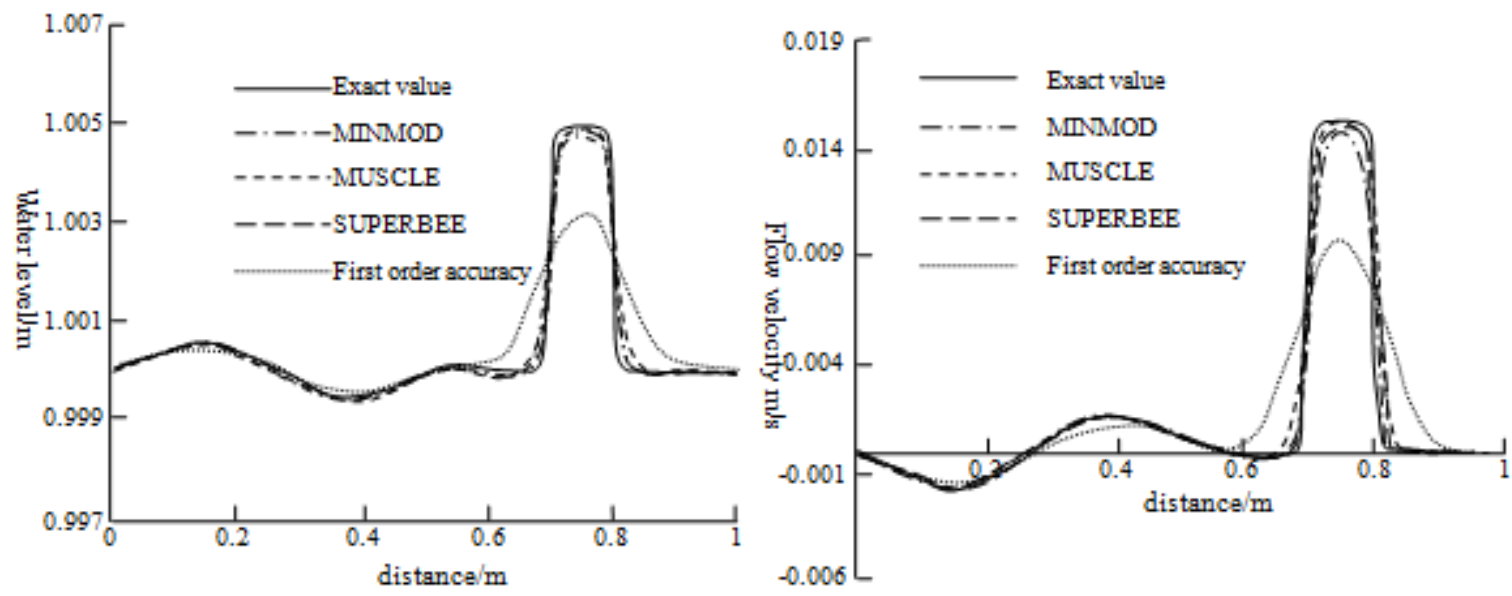

Fig.2

\section{Conclusion}

This paper is based on the Roe approximate Riemann solution, by means of MUSCL reconsitution、 TVD property flux limiter function and two-steps Hancock method, high accuracy hydraulic discrete form can be obtained. By comparing the flux limiter function in two-dimension asymmetric dam break and Leveque water wave example shows that this method is of conservative、robustness、no spurious numericalosc and high resolution shock capturing, overcome discontent entropy condition at critical flow.

\section{Acknowledgements}

This work was financially supported by the Doctoral Scientific Research Foundation of Inner Mongolia University for Nationality NO. BS325.

\section{References}

[1] Ami Harten. High resolution schemes for hyperbolic conservation laws [J]. Journal of Computational Physics, 1983, 49(3): 357:393.

[2] S F Davis, A simplified TVD finite difference scheme via artificial viscosity [J]. SIAM Journal on Scientific Computing, 1987, 8(1): 1-18.

[3]P. L. Roe. Approximate Riemann solvers, parameter vectors, and difference schemes [J]. Journal of Computational Physics, 1981, 43(2): 357-372.

[4] Hu K, Mingham C G, Causon D M. A bore-capturing finite volume method for open-channel flows. Int. J. Meth. Fluids, 1998,28:1241-1261.

[5] LEVEQUE R J. Balancing source terms and flux gradient in high-resolution Godunov method: the quasi steady wave propogation algorithm[J]. J. Comp. Phys., 1998,148:346-365. 